\title{
Juegos cooperativos y razonamiento prosocial en niños: efectos de un programa de intervención
}

\section{Cooperative games and prosocial reasoning in children: effects of an intervention program}

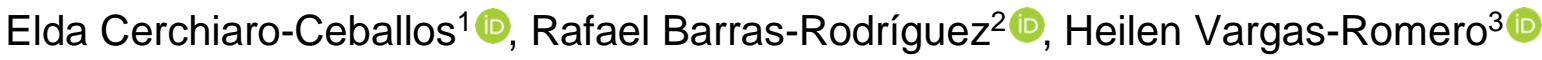

Tipología: Artículo de investigación científica y tecnológica.

Para citar este artículo: Cerchiaro-Ceballos E, Barras-Rodríguez R, Vargas-Romero H. Juegos cooperativos y razonamiento prosocial en niños: efectos de un programa de intervención. Duazary. 2019 septiembre;16(3): 40 - 53. Doi: http://dx.doi.org/10.21676/2389783X.2967

Recibido en abril 30 de 2018

Aceptado en marzo 22 de 2019

Publicado en línea en septiembre 01 de 2019

\section{RESUMEN}

Este artículo presenta los resultados de un estudio realizado en una escuela pública con niños entre siete y ocho años de edad, con el fin de determinar la influencia de los juegos cooperativos en su razonamiento prosocial. Se utilizó un diseño cuasiexperimental con grupos control y experimental, con mediciones pretest y postest a través de una escala pictórica para evaluar razonamiento prosocial en los niños participantes, antes y después de la implementación de un programa psicoeducativo basado en juegos cooperativos. Con la aplicación del programa se registra un cambio significativo en el razonamiento prosocial de los niños, manifestado en un aumento en los juicios orientado a las necesidades y estereotipado, acompañado de una disminución en el razonamiento hedonista. Los resultados muestran un avance en el desarrollo prosocial de los niños participantes en el grupo experimental, quienes alcanzan niveles de razonamiento prosocial de una mayor complejidad. De esta manera se pone a prueba el potencial de los juegos cooperativos para promover el razonamiento y la conducta prosocial de niños en contextos escolares.

Palabras clave: razonamiento prosocial; juegos cooperativos; programa de intervención; escuela primaria.

\footnotetext{
${ }^{1}$ Universidad del Magdalena. Santa Marta, Colombia. Correo: ecerchiaro@ unimagdalena.edu.co - https://orcid.org/0000-0002-6440-4659

${ }^{2}$ Universidad del Magdalena. Santa Marta, Colombia. Correo: rafaelbarrasrodriguez@ gmail.com - https://orcid.org/0000-0001-50835645

${ }^{3}$ Universidad del Magdalena. Santa Marta, Colombia. Correo: heilentatianavargasromero@ gmail.com - https://orcid.org/0000-00027769-2071
} 
Juegos cooperativos y razonamiento prosocial en niños: efectos de un programa de intervención

\section{ABSTRACT}

This article presents the results of a study made with children between seven and eight years of age, with the purpose of determining the influence of cooperative games on their prosocial judgement. It has a quasi-experimental design with control and experimental groups, with pre-test and post-test measurements through a pictorical scale to evaluate prosocial reasoning in the participating children, before and after the implementation of an intervention program based on cooperative games. The program had a significant impact on children's prosocial judgement, there was an increase in the frequency of children with needs-oriented and stereotyped reasoning, and a decrease in hedonistic judgement. Psychoeducational programs of this type could be used to positively influence the reasoning and prosocial behavior of children at these ages.

Keywords: prosocial judgement; cooperative games; intervention program; elementary school.

\section{INTRODUCCIÓN}

El estudio de conductas prosociales en niños y adolescentes ocupa en la actualidad un lugar destacado en la investigación psicológica ${ }^{1}$. Esto aparece asociado al hecho de que promover el desarrollo de habilidades prosociales se ha convertido en un objetivo de primer orden desde distintos contextos de socialización. Muchos de estos estudios se centran en la comprensión de su origen a partir de manifestaciones tempranas de altruismo y cooperación en la primera infancia $^{2}$, su desarrollo hasta la edad adulta ${ }^{3}$, la descripción de mecanismos que las hacen posibles $^{4,5}$ y factores cognitivos, motivacionales y afectivos que subyacen a estos comportamientos 6 . Los resultados que arroja este campo de la investigación sugieren que es posible afectar positivamente la manifestación de comportamientos prosociales en estas edades por la vía de intervenciones puntuales que promuevan actos solidarios, empáticos y de ayuda a los otros. En esta misma línea, este artículo pretende establecer los cambios que se producen en el razonamiento prosocial de niños y niñas entre siete y ocho años de edad como resultado de su participación en un programa psicoeducativo basado en juegos cooperativos. Esta propuesta, que ha sido poco explorada en la bibliografía reciente sobre prosocialidad, busca incidir en el desarrollo de comportamientos prosociales en los niños a partir de cambios en su razonamiento prosocial, el cual, como se sabe, se encuentra en la base de estas conductas.
El razonamiento prosocial es uno de los principales precursores de la conducta prosocial $^{7,8}$. Este atañe a la manera como una persona se enfrenta y resuelve conflictos en los que debe escoger entre satisfacer sus propios deseos y necesidades o los de otras personas, en contextos en los cuales las leyes, los castigos, la autoridad, las obligaciones formales y otros criterios externos son irrelevantes o minimizados ${ }^{9}$. El razonamiento cognitivo y el juicio moral son procesos en los que se apoya un sujeto cuando se enfrenta a situaciones como estas que involucran a uno o varios individuos con algún tipo de necesidad, en las que los valores, los intereses, las creencias, las emociones y la capacidad para la toma de perspectiva de alguien son puestos a prueba ${ }^{10}$.

Nancy Eisenberg ${ }^{7}$ es una de las autoras que han logrado consolidar un corpus empírico sólido sobre el razonamiento prosocial. En sus investigaciones va más allá de los planteamientos de Kolhberg ${ }^{8}$ sobre desarrollo moral y aborda su estudio a través del razonamiento y la conducta moral prosocial. Esta última involucra acciones voluntarias que benefician a otra persona o grupo de personas, como ayudar, compartir o consolar, sin que medie algún tipo de recompensa externa para quien las realiza ${ }^{6}$. Así pues, los comportamientos prosociales comprenden una amplia variedad de conductas que incluyen el compartir, el apoyo o la ayuda, la cooperación, entre otras acciones positivas dirigidas hacia los demás ${ }^{11}$. 
En el estudio del razonamiento prosocial se han utilizado dilemas morales prosociales que involucran la conducta prosocial. Se trata de situaciones hipotéticas que plantean conflictos entre las necesidades y deseos de diferentes actores, e indagan acerca de si sería correcto ayudar, dar o compartir con otros a expensas de sus propias metas y deseos ${ }^{9}$. Las respuestas de los niños ante estos dilemas revelan una tendencia hacia el razonamiento moral prosocial, cuyo desarrollo es progresivo y está articulado a su desarrollo cognitivo ${ }^{3,9}$. Este proceso se caracteriza por cambios cualitativos en la manera como el sujeto razona frente a diversas situaciones que requieren un actuar prosocial, en las cuales debe decidir cómo proceder sin que exista ningún tipo de presión más que sus principios morales y prosociales.

A partir de estas investigaciones, Eisenberg y sus colegas identifican estos cambios en el razonamiento prosocial y los organizan en seis tipos o niveles, en función del desarrollo de la capacidad empática y de la progresiva consideración que hace el individuo de las necesidades de los demás ${ }^{12}$. Los juicios que los sujetos hacen sobre sus acciones prosociales se reflejan en estos niveles, los cuales se encuentran directamente relacionados con la edad y siguen una tendencia progresiva ${ }^{13}$. No obstante, aunque existe una estrecha correlación entre la edad y el razonamiento prosocial, los niveles propuestos no constituyen estructuras jerárquicas e integradas, como tampoco puede considerarse esta secuencia completamente invariante $y$ universal $^{12}$, dado que puede variar según situaciones y circunstancias de la vida de los individuos.

Como puede observarse en la Tabla 1, en el primer nivel los juicios se basan en una orientación hedonista, centrada en la propia persona, según la cual el beneficio personal prima sobre la ayuda que se brinde a otros, mientras que en el segundo nivel hay una orientación a las necesidades de las otras personas. Sin embargo, a pesar de esta tendencia de ayuda al otro, no existe aún una toma de perspectiva, ni motivaciones morales, o emociones positivas que guíen la conducta prosocial. 
Tabla 1. Niveles de razonamiento prosocial según Eisenberg

\begin{tabular}{|c|c|}
\hline $\begin{array}{c}\text { Tipo de razonamiento } \\
\text { prosocial }\end{array}$ & Características \\
\hline $\begin{array}{l}\text { Hedonista } \\
\text { (preescolar e inicios de } \\
\text { la escuela primaria) }\end{array}$ & $\begin{array}{l}\text { Las necesidades propias o de las personas allegadas se anteponen a las necesidades de } \\
\text { los otros. Obtener algún tipo de beneficio para sí, o para las personas cercanas, es uno } \\
\text { de los aspectos que tienen mayor peso en el momento de decidir ayudar o no hacerlo. } \\
\text { Ejemplo: si comparto mis galletas con Pedro, me quedaré sin comer. }\end{array}$ \\
\hline $\begin{array}{c}\text { Orientado a las } \\
\text { necesidades de los } \\
\text { otros } \\
\text { (preescolar y escuela } \\
\text { primaria) }\end{array}$ & $\begin{array}{l}\text { Existe un interés por ayudar generado por la necesidad manifiesta de otra persona. Se } \\
\text { basa en una empatía primitiva, en donde no hay una toma de perspectiva del otro, ni } \\
\text { motivaciones morales, o emociones positivas que guíen la conducta prosocial. } \\
\text { Ejemplo: compartiré mi merienda con Ana porque tiene hambre. }\end{array}$ \\
\hline $\begin{array}{c}\text { Centrado en la } \\
\text { aprobación de los } \\
\text { demás } \\
\text { (escuela primaria e } \\
\text { inicios de secundaria) }\end{array}$ & $\begin{array}{l}\text { Se inclina hacia la realización de acciones prosociales. No obstante, el interés de } \\
\text { ayudar se basa en recibir como recompensa una valoración positiva de personas } \\
\text { representativas (suelen ser los padres y maestros para los niños). Ejemplo: si ayudo a } \\
\text { Camila con su tarea, la maestra me felicitará. }\end{array}$ \\
\hline $\begin{array}{l}\text { Estereotipado } \\
\text { (escuela primaria y } \\
\text { secundaria) }\end{array}$ & $\begin{array}{l}\text { Las concepciones y creencias que tenga el individuo sobre lo que es bueno o malo, en } \\
\text { relación con las personas y las acciones, son las que determinan y justifican el actuar } \\
\text { prosocial. Ejemplo: ayudé a Juan a cargar su bolso pesado porque ayudar es bueno. }\end{array}$ \\
\hline $\begin{array}{l}\text { Orientación empática } \\
\text { (final de la escuela } \\
\text { primaria e inicios de la } \\
\text { secundaria) }\end{array}$ & $\begin{array}{l}\text { Las acciones prosociales de la persona están motivadas por la preocupación y } \\
\text { compasión que se tiene por el otro que necesita ayuda. Ejemplo: compartí con Andrea } \\
\text { mis dulces porque me preocupaba que ella no comiera. }\end{array}$ \\
\hline $\begin{array}{l}\text { Afecto internalizado } \\
\text { (final de la escuela } \\
\quad \text { secundaria) }\end{array}$ & $\begin{array}{l}\text { La persona actúa basada en las consecuencias que su actuación pueda generar en sí } \\
\text { misma y en los otros, teniendo siempre como referencia sus valores internalizados. } \\
\text { Ejemplo: consolé a mi amigo cuando lloraba para que se sintiera mejor, y eso me hace } \\
\text { sentir bien. }\end{array}$ \\
\hline
\end{tabular}

Fuente: elaboración propia basada en Eisenberg ${ }^{12,13}$.

En el tercer nivel, la intención de ayudar se basa en recibir aprobación y aceptación de otros, a diferencia del cuarto nivel, en el que los juicios están basados en estereotipos sobre lo que significa hacer el bien y el mal. El quinto nivel se caracteriza por una orientación autorreflexiva y empática que comprende la preocupación, el cuidado y la toma de perspectiva de los demás. Por último, en el sexto nivel existe una internalización de los afectos vinculados a la autoestima, de las leyes, normas, deberes y responsabilidades, así como de los tipos abstractos de razonamiento sobre la sociedad, los derechos, la justicia y la igualdad ${ }^{13-16}$.

Estudios longitudinales realizados en diferentes países sobre el desarrollo del razonamiento prosocial vinculan estos niveles a ciertas edades $^{13}, 16-18$. De este modo se ha llegado a establecer que el razonamiento de tipo hedonista es característico en los niños en edad preescolar, pero en la escuela primaria disminuye considerablemente y empieza a ser reemplazado en algunos casos por un razonamiento orientado 
a la aprobación, que puede extenderse hasta la adolescencia, o en otros casos por un razonamiento prosocial orientado a las necesidades de los otros, que comienza a declinar al inicio de la adolescencia. El razonamiento de tipo estereotipado se consolida entre los nueve y 12 años, para luego tender a disminuir. La orientación empática y el afecto internalizado son característicos de la adolescencia y la edad adulta.

Algunos de estos estudios demuestran correlación positiva alta entre el razonamiento prosocial y las conductas prosociales. Esto significa que cuanto mayor sea el primero, mayor será la probabilidad de que se muestren las segundas ${ }^{14,19}$, sobre todo aquellas que tienen un alto costo como donar, compartir, actividades de voluntariado, etc. De igual manera, se ha encontrado que el razonamiento prosocial es uno de los predictores más importantes de las acciones prosociales, junto con la simpatía ${ }^{7,20}$, al mismo tiempo que se reportan niveles bajos de razonamiento prosocial asociados con escasas manifestaciones de prosocialidad, acciones prosociales de bajo costo, e incluso con comportamientos agresivos ${ }^{18}$.

Este tipo de evidencia empírica apoya la hipótesis de este estudio, según la cual la aplicación de estrategias que busquen el desarrollo del razonamiento prosocial podría tener un impacto significativo en la manifestación de conductas prosociales. No obstante, pese a encontrarse un número considerable de estrategias dirigidas a fomentar la prosocialidad en la escuela, son muy pocos los programas de intervención realizados cuyos esfuerzos estén orientados a promover el desarrollo del razonamiento prosocial, en especial con niños en edades escolares.

La práctica de comportamientos cooperativos ha sido uno de los medios utilizados para optimizar conductas prosociales en niños y adolescentes ${ }^{21}$ a través de programas educativos en contextos escolares. La cooperación es un tipo de comportamiento prosocial en el que dos o más individuos se ayudan y colaboran mutuamente para conseguir el mismo fin $^{22}$. Propicia el establecimiento de relaciones positivas entre las personas, quienes, utilizando las herramientas necesarias y/o disponibles, podrán alcanzar fines grupales o individuales, solo bajo la condición de que los otros logren alcanzar los suyos. Tener habilidades básicas de cooperación es fundamental para que las relaciones humanas sean más productivas en la escuela, el trabajo o en cualquier $\operatorname{sitio}^{23,24}$, en tanto que la eficacia y el rendimiento individual y colectivo se ven favorecidos por la convergencia de intereses y acciones. Por consiguiente, si se promueven en los niños habilidades básicas de cooperación, las relaciones sociales en las que ellos participen a lo largo de su vida se verán beneficiadas.

Por lo anterior, los juegos cooperativos han sido utilizados como una estrategia fundamental para influir en la conducta prosocial de los niños ${ }^{23,25,26}$, dado que permiten desarrollar habilidades de ayuda, cuidado por el otro y cooperación. Se trata de actividades lúdicas cuya esencia se desliga totalmente de un ejercicio competitivo, para brindar a los jugadores un ambiente de interacción libre de expectativas de logro o de fracaso, que provoca el disfrute del juego en sí mismo al eliminar la posibilidad de un resultado final que coloca a los participantes como ganadores versus perdedores ${ }^{27}$.

El estudio que aquí se presenta consiste en el desarrollo de un programa psicoeducativo con niños escolares, basado en juegos cooperativos, que busca producir cambios en el razonamiento prosocial de estos sujetos y de esta manera favorecer comportamientos prosociales. No se tiene referencia de otros estudios que persigan el mismo propósito. Investigaciones previas que utilizan juegos cooperativos como estrategia de intervención analizan los efectos producidos en las esferas socioafectivas y comportamentales $^{23,28-31}$, en términos de la disminución de conductas disruptivas y antisociales $^{32}$, la manifestación de conductas prosociales $^{29,30}$, el desarrollo de habilidades sociales $^{33}$, la capacidad para resolver problemas $^{32}$, la empatía y la toma de perspectiva $^{28}$. Sin embargo, estos trabajos no 
Juegos cooperativos y razonamiento prosocial en niños: efectos de un programa de intervención

consideran el impacto que los juegos cooperativos puedan tener en el desarrollo cognitivo y moral de los niños. Es así como, en contraste con dichas propuestas, esta investigación busca determinar de qué manera contribuye un programa de juegos cooperativos desarrollado en una escuela pública en el razonamiento moral prosocial de niños entre siete y ocho años.

\section{MATERIALES Y MÉTODOS}

\section{Tipo de estudio}

Se utiliza un diseño cuasiexperimental de grupo experimental y grupo control, con mediciones pretest y postest en ambos grupos ${ }^{34}$ para evaluar el razonamiento prosocial de los niños participantes, antes y después de la implementación de un programa de intervención basado en juegos cooperativos.

\section{Población y muestra}

La muestra fue escogida de una población de 85 niños y niñas de entre siete y ocho años, matriculados en una escuela pública de la ciudad de Santa Marta (Colombia) y quedó conformada por 59 participantes: 34 niños y 25 niñas $(\mathrm{M}=7,98 ; \mathrm{DT}=0,13)$, de tres aulas de segundo grado de básica primaria. La selección se hizo mediante un muestreo por conveniencia teniendo en cuenta dos criterios de inclusión: la edad (mayores de siete años y un día y no mayores de ocho años 11 meses de edad) y la autorización firmada por los padres para participar en la investigación. Los participantes pertenecen a familias que se ubican mayoritariamente en estratos socioeconómicos uno y dos (69\%). Casi la mitad de los padres de familia completó sus estudios de bachillerato (54\%), y solo $18 \%$ tiene título profesional.

Los participantes hacían parte de cuatro cursos del grado tercero de primaria. La asignación de estos en los grupos control y experimental se hizo de manera aleatoria: 29 en el primero (20 niños y 9 niñas) y 30 en el segundo (14 niños y 16 niñas). Todos los niños fueron sometidos a una medición pretest y postest una semana antes y una semana después de la intervención, respectivamente.

\section{Instrumentos}

Para evaluar el nivel de razonamiento prosocial en los participantes se utilizó la Escala Pictórica de Razonamiento Prosocial ${ }^{17}$. Este instrumento es una adaptación gráfica de una versión verbal $^{35}$, inspirada en la escala Medida Objetiva de Razonamiento Moral Prosocial ${ }^{36}$, diseñada para adolescentes y adaptada a sujetos hispanohablantes ${ }^{37}$.

La escala propone una serie de dilemas morales que son presentados a los niños como situaciones problemáticas a las cuales deben encontrar alguna solución. Se trata de cinco historias que son narradas al niño, en las que se debe emitir un juicio frente a lo que el personaje de la historia debería hacer. Por su contenido moral, estos dilemas ponen a prueba los valores y las concepciones de "bueno" o "malo" que cada niño tiene a través de las soluciones que plantea. A partir de la respuesta se califica la acción prosocial que el niño considera correcta, y seguidamente se indaga sobre aquello que el personaje de la historia pensó en el momento de realizar dicha acción. Con este propósito se presentan al niño cuatro tarjetas con dibujos, que representan distintos tipos de razonamiento que el personaje de la historia pudo haber realizado. Se pide entonces al niño que organice de manera jerárquica las tarjetas, con el fin de valorar el tipo de razonamiento prosocial que subyace a la escogencia de un determinado comportamiento prosocial.

La escala permite crear un perfil del razonamiento prosocial de cada niño. Las respuestas verbales, junto con la organización de las tarjetas, son puntuadas según el nivel del razonamiento prosocial que expresen (Tabla 1). Los puntajes de cada nivel son promediados, y de este modo se obtienen cuatro puntajes finales a partir de los cuales se puede establecer un perfil individual del razonamiento prosocial. 
La consistencia interna de la escala oscila entre $\alpha 0,50$ y $\alpha 0,56$, valores que, aunque no son altos, resultan aceptables teniendo en cuenta que va dirigida a niños pequeños, además de que involucra un constructo difícil de evaluar en estas edades.

La aplicación de la escala se hizo de manera individual en la institución educativa a la que asisten los niños, para lo cual se dispuso de una sala con las condiciones de privacidad y aislamiento requeridas.

\section{Intervención}

El programa se desarrolló en las instalaciones de la institución educativa donde los niños se encuentran matriculados, en 18 sesiones de dos horas cada una, con una frecuencia de tres sesiones semanales. Se cumplieron tres momentos o fases:

Fase de sensibilización: durante la primera sesión se presentó el programa a los niños, informándoles sobre los objetivos y las actividades que se iban realizar. El propósito era crear sensibilidad sobre la importancia del trabajo en equipo y motivarlos a participar de manera comprometida en el desarrollo de las actividades.
Fase de aplicación: se realizaron 16 sesiones centradas en diferentes juegos cooperativos. Desde escenarios distintos, cada juego propuso a los niños una tarea determinada que solo podían cumplir con éxito si contaban con la participación solidaria de otros compañeros (Figura 1). Cada sesión comenzaba con una descripción del juego, su propósito y las reglas para su aplicación. Los niños, organizados en pequeños grupos, eran orientados en la realización de las actividades propias del juego. Al final de la sesión, se disponía de media hora como espacio de reflexión para explorar las percepciones de los participantes acerca del juego desarrollado y lo aprendido a través de este. Por último, se planteaban conclusiones a partir de la discusión generada. Los niños del grupo control no participaron de estas actividades; durante esta fase ellos continuaron con las actividades académicas a cargo de sus maestros.

Fase de cierre: en la última sesión se realizó una actividad de integración con los niños tanto del grupo control como del grupo experimental, con el propósito de hacerles un reconocimiento por su participación en el programa.

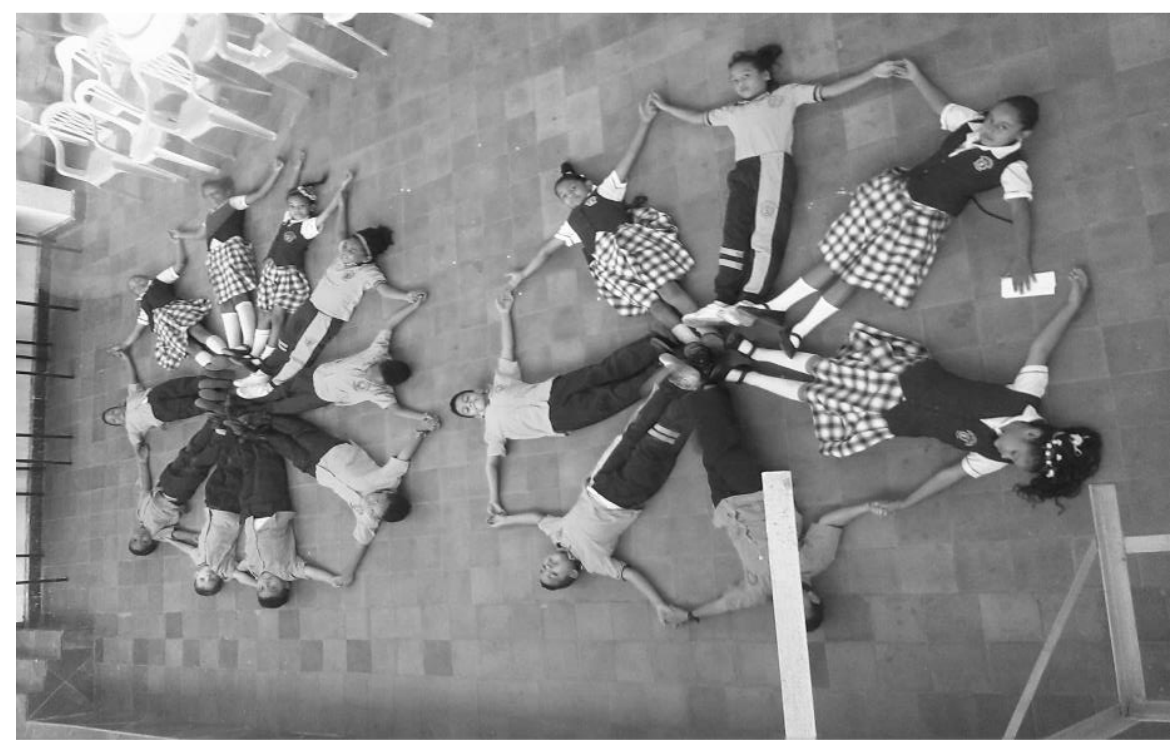

Figura 1. Niños participando en la sesión 9 


\section{Análisis de los datos}

El análisis se dirige a establecer diferencias en el nivel de razonamiento prosocial manifestado por los participantes tanto en el grupo experimental como en el grupo control, antes y después de la intervención. Mediante la prueba estadística no paramétrica U de Mann-Withney para muestras independientes se realiza la comparación entre los dos grupos, a partir de las medianas de las puntuaciones directas obtenidas para cada tipo de razonamiento moral prosocial en las mediciones pretest y postest. Se presentan además estadísticos descriptivos para comparar la frecuencia de los razonamientos prosociales en ambos grupos, tanto en pretest como en postest. Para dicho fin, se escogió el tipo de razonamiento prosocial con mayor puntuación por cada niño, comprendiendo que este caracteriza su juicio prosocial.

\section{Declaración sobre aspectos éticos}

Teniendo en cuenta los principios y normas éticas para la investigación establecidos en la declaración de Helsinki de la Asociación Médica Mundial que recoge la Resolución 8430 de 1993 del Ministerio de Salud de Colombia ${ }^{39}$, la participación de los niños fue autorizada por los padres mediante consentimiento informado, en el que permiten además el uso exclusivo de imágenes y videos en los que aparecen los niños para fines académicos y de investigación. Asimismo, la información suministrada por los niños, sus representantes legales y las directivas de la institución fue manejada bajo el criterio de confidencialidad. De igual manera, la participación voluntaria de la institución educativa se formalizó también a través de consentimiento informado suscrito por la directora.

\section{RESULTADOS}

En la Tabla 2 se muestran los resultados que arroja la prueba estadística U de Mann-Whitney en relación con las diferencias encontradas en las medianas entre el grupo control y el grupo experimental, según el tipo de razonamiento. Los datos obtenidos indican que no existen discrepancias significativas entre los dos grupos en la primera medición (pretest), mientras que en la segunda (postest) se constatan diferencias estadísticamente significativas en el razonamiento prosocial de ambos grupos, en específico en los tipos de razonamiento hedonista $(\mathrm{U}=129,500 ; \mathrm{P}<0,001)$, orientado a las necesidades $\quad(\mathrm{U}=176,000 ; \quad \mathrm{P}<0,001) \quad \mathrm{y}$ estereotipado ( $\mathrm{U}=191,500 ; \mathrm{P}<0,001)$. 
Tabla 2. Puntuaciones globales obtenidas en los dos grupos en el pretest y el postest

\begin{tabular}{|c|c|c|c|}
\hline $\begin{array}{c}\text { Tipo de razonamiento } \\
\text { prosocial }\end{array}$ & Prueba & Pretest & Postest \\
\hline \multirow{4}{*}{ Hedonista } & U de Mann-Whitney & 402,000 & 129,500 \\
\cline { 2 - 4 } & Mediana grupo control & 11 & 13 \\
\cline { 2 - 4 } & Mediana grupo experimental & 10 & 7 \\
\cline { 2 - 4 } & Sig. asintótica (bilateral) & 0,616 & $0,000^{*}$ \\
\hline \multirow{4}{*}{ Orientado a la aprobación } & U de Mann-Whitney & 432,500 & 267,000 \\
\cline { 2 - 4 } & Mediana grupo control & 11 & 8 \\
\cline { 2 - 4 } & Mediana grupo experimental & 11 & 7 \\
\cline { 2 - 4 } & Sig. asintótica (bilateral) & 0,854 & 0,010 \\
\hline \multirow{4}{*}{ Orientado a las necesidades } & U de Mann-Whitney & 423,000 & 176,000 \\
\cline { 2 - 4 } & Mediana grupo control & 7 & 10,5 \\
\cline { 2 - 4 } & Mediana grupo experimental & 7 & 14 \\
\cline { 2 - 4 } & Sig. asintótica (bilateral) & 0,854 & $0,000^{*}$ \\
\hline \multirow{3}{*}{ Estereotipado } & U de Mann-Whitney & 414,000 & 191,500 \\
\cline { 2 - 4 } & Mediana grupo control & 11 & 8,5 \\
\cline { 2 - 4 } & Mediana grupo experimental & 11 & 12 \\
\cline { 2 - 4 } & Sig. asintótica (bilateral) & 0,749 & $0,000 *$ \\
\hline \multirow{4}{*}{$*$ P<0,001 } & & & \\
\hline
\end{tabular}

En la Tabla 3 se presentan las frecuencias y los porcentajes alcanzados por el grupo control (GC) y el grupo experimental (GE) en cada tipo de razonamiento prosocial, tanto en el pretest como en el postest. En el pretest no se registran diferencias significativas entre los dos grupos, lo cual constituye un indicador de la equivalencia de estos. En esta primera medición el razonamiento prosocial de tipo hedonista reporta una mayor frecuencia entre los niños evaluados, con $36,7 \%$ para el grupo control y $37,9 \%$ en el grupo experimental, en tanto los tipos de razonamiento estereotipado y orientado a las necesidades comparten un $23,3 \%$ en el grupo control. Caso similar ocurre en el grupo experimental, en el cual estos dos tipos de razonamiento alcanzan 20,7\%. Finalmente, el tipo orientado a la aprobación llega en el grupo control a un $16,7 \%$ y en el grupo experimental hasta $20,7 \%$. 
Tabla 3. Diferencias en razonamiento prosocial de grupo control y grupo experimental en pretest y postest

\begin{tabular}{|c|c|c|c|c|c|}
\hline \multirow{2}{*}{\multicolumn{2}{|c|}{ Tipo de razonamiento prosocial }} & \multicolumn{2}{|c|}{ Pretest } & \multicolumn{2}{|c|}{ Postest } \\
\hline & & \multirow{2}{*}{$\begin{array}{ll}\mathrm{GC} & \\
& 11\end{array}$} & \multirow{2}{*}{$\begin{array}{ll}\mathrm{GE} & \\
& 11\end{array}$} & \multirow{2}{*}{$\begin{array}{ll}\mathrm{GC} & \\
& 16\end{array}$} & \multirow{2}{*}{$\begin{array}{l}\mathrm{GE} \\
\end{array}$} \\
\hline \multirow{2}{*}{ Hedonista } & Número & & & & \\
\hline & Porcentaje & $36,7 \%$ & $37,9 \%$ & $53,3 \%$ & $3,4 \%$ \\
\hline \multirow{2}{*}{$\begin{array}{l}\text { Orientado a la } \\
\text { aprobación }\end{array}$} & Número & 5 & 6 & 4 & 1 \\
\hline & Porcentaje & $16,7 \%$ & $20,7 \%$ & $13,3 \%$ & $3,4 \%$ \\
\hline \multirow{2}{*}{$\begin{array}{l}\text { Orientado a las } \\
\text { necesidades }\end{array}$} & Número & 7 & 6 & 5 & 15 \\
\hline & Porcentaje & $23,3 \%$ & $20,7 \%$ & $16,7 \%$ & $51,7 \%$ \\
\hline \multirow{2}{*}{ Estereotipado } & Número & 7 & 6 & 5 & 12 \\
\hline & Porcentaje & $23,3 \%$ & $20,7 \%$ & $16,7 \%$ & $41,5 \%$ \\
\hline \multirow[t]{2}{*}{ Total } & Número & 30 & 29 & 30 & 29 \\
\hline & Porcentaje & $100,00 \%$ & $100,00 \%$ & $100,00 \%$ & $100,00 \%$ \\
\hline
\end{tabular}

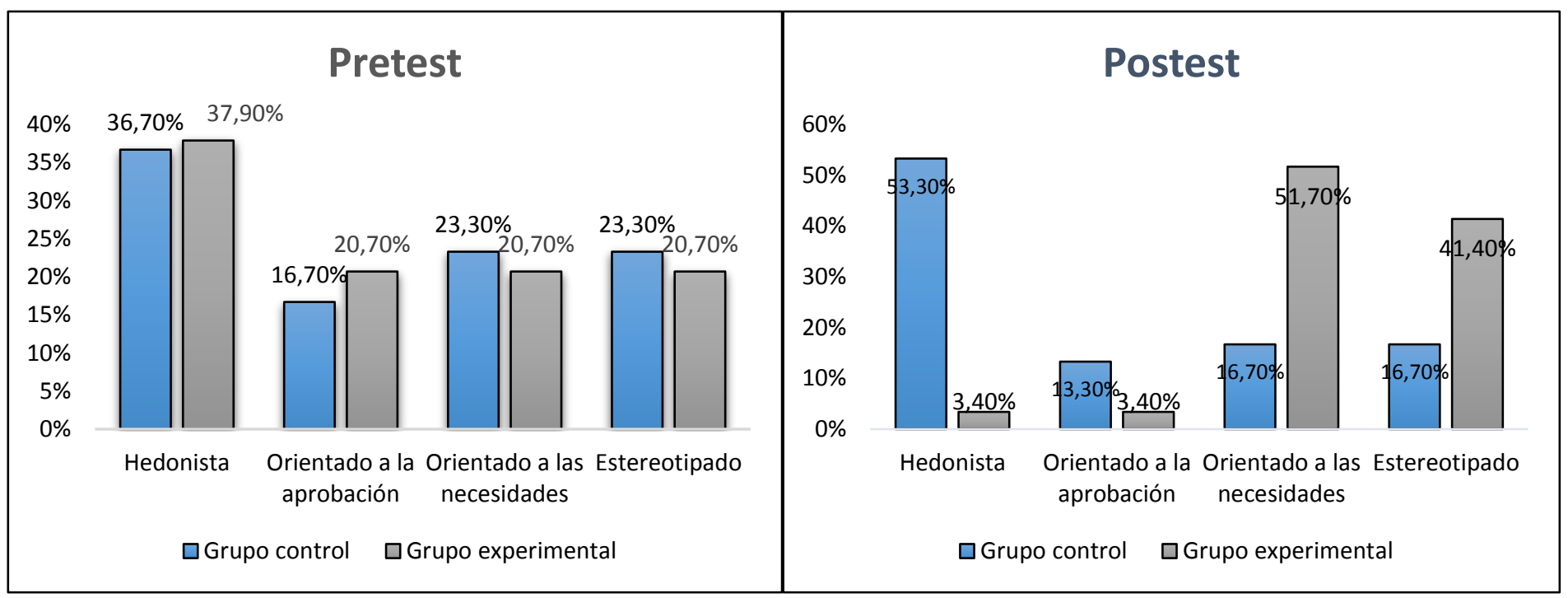

Figura 2. Resultados pretest y postest, grupo control y grupo experimental

En la medición postest (Figura 2), el grupo experimental presenta cambios importantes en la frecuencia que registran los tipos de razonamiento prosocial en comparación con el pretest. El 51,7\% de los niños de dicho grupo se ubica en el tipo orientado a las necesidades, siendo este el más frecuente de todos, a diferencia del pretest, en donde el hedonista fue el de mayor frecuencia. En este mismo grupo, el razonamiento estereotipado se muestra en $41,4 \%$, mientras que los tipos de razonamiento orientado a la aprobación y hedonista alcanzan un porcentaje de $3,4 \%$, llegando a ser los de menor frecuencia en esta última medición. De manera general, en la evaluación postest el grupo mencionado registra una disminución significativa en las puntuaciones obtenidas por los niños en los tipos de razonamiento prosocial hedonista y orientado a la aprobación, asociada a un aumento en las puntuaciones en los tipos orientado a las necesidades y estereotipado.

En cuanto al grupo control, en el postest muestra un incremento en el porcentaje de niños que se ubican en el razonamiento de tipo hedonista 
(53,3\%), al lado de una disminución en los porcentajes correspondientes a los tipos de razonamiento orientado a la aprobación $(13,3 \%)$, orientado a las necesidades (16,7\%) y estereotipado (16,7\%), en relación con el pretest.

\section{DISCUSIÓN}

El propósito de este estudio fue determinar cómo la participación de niños entre siete y ocho años en actividades prosociales de ayuda $y$ cooperación a través de un programa psicoeducativo en la escuela produce cambios en su razonamiento prosocial. Los resultados indican que el programa desarrollado tuvo un impacto positivo y significativo en el razonamiento prosocial de estos niños. Antes de la intervención los participantes manifiestan un razonamiento prosocial propio del nivel preescolar, de acuerdo con la tipología establecida por Eisenberg y sus colaboradores ${ }^{16}$, mientras que, después de finalizado el programa, se comprueba un aumento en la frecuencia de razonamientos de tipo orientado a las necesidades y estereotipado en los participantes del grupo experimental. Esto significa que la mayoría de los niños reflejaron cambios en su emisión de juicios sobre actos morales prosociales, pasando de considerar el beneficio propio como aspecto central en la toma de decisiones a tener en cuenta la necesidad manifiesta de otra persona. Esta apreciación que hace el niño está fundada en un sentimiento primario de empatía o en sus concepciones y creencias sobre lo que es bueno o malo para justificar su actuación prosocial. Si bien otros estudios han demostrado que el nivel hedonista disminuye con la edad ${ }^{16}$, en esta investigación este cambio en el tipo de razonamiento en los niños se encuentra asociado a su participación en el programa de juegos cooperativos. Esto se corrobora con las diferencias encontradas en las mediciones de los grupos control y experimental, en las cuales se registran pocas variaciones entre los resultados pretest y postest en los niños del grupo control.

Estos resultados coinciden con los hallazgos de Lemos y Richaud de Minzi ${ }^{17}$, quienes encuentran que niños de siete años se ubican en niveles hedonistas, mientras que los niños de ocho años dan muestras de un razonamiento estereotipado y orientado a las necesidades. En nuestros datos este último aspecto se observa únicamente con los participantes del grupo experimental en la medición postest, lo cual contrasta con la evidencia presentada ${ }^{14}$ sobre la aparición del nivel de razonamiento estereotipado entre los nueve y 11 años de edad y su disminución progresiva a medida que se acerca a la adolescencia.

La relación entre razonamiento prosocial y conductas cooperativas explorada en este estudio se constata en otras investigaciones que muestran evidencia a favor de una relación positiva entre estas dos variables, como sucede en el trabajo realizado con niños de 10 años cuyo razonamiento prosocial muestra correlación positiva con las acciones cooperativas espontáneas que realizan con sus madres ${ }^{39}$. De igual manera, en un estudio de carácter longitudinal con niños de seis años se encuentra que el razonamiento prosocial es un predictor de las conductas de cooperación y de compartir, aunque en niveles más bajos que la simpatía y la culpa $^{18}$.

Es importante destacar el componente metacognitivo incorporado al programa desarrollado, que estuvo orientado a la toma de conciencia sobre valores como solidaridad, compañerismo, bondad, entre otros, a través de los espacios de reflexión creados al finalizar cada sesión del programa, los cuales permitían a los niños extraer conclusiones de la actividad realizada y comprender la importancia del trabajo en equipo, condición necesaria para alcanzar el éxito en cada juego. Este componente metacognitivo también ha sido trabajado en otros programas de juegos cooperativos ${ }^{23,32}$, con resultados importantes en la expresión de emociones positivas, la comunicación intragrupal, la metacognición y el desarrollo moral. Sin embargo, hace falta un análisis más profundo del papel que este ejercicio de metacognición vinculado a la práctica de juegos 
cooperativos puede tener en el desarrollo del razonamiento moral prosocial.

Los resultados de este estudio son relevantes en dos sentidos. De una parte, muestran un progreso en el razonamiento moral prosocial de los niños participantes, quienes alcanzan modalidades de razonamiento de una mayor complejidad que se acercan a una toma de perspectiva y a sentimientos iniciales de empatía con el otro. Por otro lado, en tanto que los cambios descritos en el razonamiento prosocial de los niños se relacionan con su participación en el programa, se demuestra el potencial de los juegos cooperativos como un medio eficaz para promover el desarrollo prosocial de los niños, a partir de la posibilidad que ofrecen para la adopción de comportamientos más positivos, en lugar de conductas competitivas, agresivas y antisociales, al tiempo que se potencia el proceso de adopción de perspectivas y se construye un conocimiento de sí mismo y de los demás ${ }^{8,15}$. La efectividad de los juegos cooperativos ha sido probada además para fomentar la empatía y la toma de perspectiva en niños en edades escolares, ${ }^{26,27}$ aspectos que se encuentran en la base de razonamientos prosociales que parten de reconocer las necesidades de los otros.

Para futuras investigaciones se sugiere establecer relación entre tipo de razonamiento prosocial y conducta prosocial de los niños a partir de los cambios que se registren después de una intervención de esta naturaleza. Además, se podría ampliar el espectro de factores vinculados a la prosocialidad y evaluar también variables como la empatía y la toma de perspectiva, con el fin de comprender mejor el impacto que tienen los juegos cooperativos en cada una de las áreas mencionadas. Asimismo, conviene hacer un seguimiento (follow up) un tiempo después de haber finalizado la intervención para determinar la consistencia del cambio operado en el nivel de razonamiento prosocial de los niños.

\section{DECLARACIÓN SOBRE CONFLICTOS DE INTERESES}

Los autores de la presente investigación manifiestan no tener conflictos de intereses.

\section{CONTRIBUCIÓN DE LOS AUTORES}

Primer autor: revisión bibliográfica y elaboración del manuscrito.

Segundo autor: trabajo de campo, diseño metodológico y análisis estadístico.

Tercer autor: trabajo de campo, construcción base de datos y redacción.

\section{REFERENCIAS BIBLIOGRÁFICAS}

1. Eisenberg N, Spinrad TL, Knafo-Noam A. Prosocial Development, Handbook of Child Psychology and Developmental Science. Sexta edición. Socioemotional Processes. Nueva York: John Wiley \& Sons, Inc; 2015.

2. Warneken F, Tomasello $\mathrm{M}$. The roots of human altruism. British Journal of Psychology. 2009;100(3):455-471.

3. Eisenberg N, Zhou Q, Spinrad TL, Valiente C, Fabes R A, Liew J. Relations among positive parenting, children's effortful control, and externalizing problems: A three-wave longitudinal study. Child Development. 2005;76(5):1055-1071.

4. Eisenberg N, Cumberland A, Guthrie I, Murphy B, Shepard S. Age changes in prosocial responding and moral reasoning in adolescence and early adulthood. Journal of Research on Adolescence. 2005;15(3): 235-260.

5. Kato-Shimizu M, Onishi K., Kanazawa T, Hinobayashi T. Preschool children's behavioral tendency toward social indirect reciprocity. PLoS One. 2013;8(8):1-10. 
6. Caprara G, Alessandri G, Eisenberg N. Prosociality: The contribution of traits, values, and self-efficacy beliefs. Journal of Personality and Social Psychology. 2012;102(6):1289-1303.

7. Eisenberg N, Zhou Q, Koller, S. Brazilian adolescents' prosocial moral judgment and behavior: Relations to sympathy, perspective taking, gender-role orientation, and demographic characteristics. Child development. 2001;72(2):518-534.

8. Kohlberg L. Psicología del desarrollo moral. Segunda edición. Bilbao: Desclée de Brouwer; 1992.

9. Eisenberg-Berg N, Hand M. The relationship of preschoolers' reasoning about prosocial moral conflicts to prosocial behavior. Child development. 1979;50(2):356-363.

10. Carlo G. Handbook of moral development Care-based and altruistically-based morality. Handbook of moral development. Mahwah: Lawrence Erlbaum; 2006.

11. Caprara GV, Steca P, Zelli A, Capanna, C. A new scale for measuring adults' prosocialness. European Journal of Psychological Assessment. 2005;21(2):77-89.

12. Eisenberg N. The Development of Prosocial Behavior. Primera edición. Estados Unidos: Academic Press; 1982.

13. Eisenberg N, Lennon R, Roth K. Prosocial development in childhood: A longitudinal study. Developmental Psychology. 1983;(19):846-855.

14. Eisenberg N, Carlo G, Murphy B, Court P. Prosocial development in late adolescence: a longitudinal study. Child development. 1995;66(4):1179-1197.

15. Siegler R, Saffran J, Eisenberg N, De Loache, J, Gershoff E. How children develop. Quinta edición. Nueva York: Worth Publishers; 2017.
16. Eisenberg N, Shell R, Pasternack J, Lennon, R, Beller R, Mathy R. Prosocial development in middle childhood: A longitudinal study. Developmental Psychology. 1987;23(5):712-718.

17. Lemos V, Richaud de Minzi M. Construcción de un instrumento para evaluar el razonamiento prosocial en niños de 7 y 8 años: una versión pictórica. Universitas Psychologica. 2010;9(3):879-891.

18. Malti T, Ongley SF, Peplak J, Chaparro MP, Buchmann M, Zuffianò A, et al. Children's Sympathy, Guilt, and Moral Reasoning in Helping, Cooperation, and Sharing: A 6-Year Longitudinal Study. Child Development. 2016;87(6):1783-95.

19. Kumru A, Carlo G, Mestre MV, Samper P. Prosocial moral reasoning and prosocial behavior among Turkish and Spanish adolescents. Social Behavior and Personality: an international journal. 2012;40(2):205-214.

20. Tur A, Llorca A, Malonda E, Samper P, Mestre M. Empatía en la adolescencia. Relaciones con razonamiento moral prosocial, conducta prosocial y agresividad. Acción Psicológica. 2016;13(2):3-14.

21. Perinat A. Psicología del desarrollo: un enfoque sistémico. Tercera edición. Barcelona: Editorial UOC; 2007.

22. Jaeggi AV, Burkart JM, Van Schaik, CP. On the psychology of cooperation in humans and other primates: combining the natural history and experimental evidence of prosociality. Philosophical Transactions of the Royal Society B: Biological Sciences. 2010;365(1553):27232735.

23. Orlick, T. Cooperative games and sports: Joyful activities for everyone. Segunda edición. Champaign: Editorial Human Kinetics; 2006.

24. Velázquez C. El aprendizaje cooperativo en Educación Física. La formación de los grupos y 
su influencia en los resultados. Tándem. 2012;(39):75-84.

25. Street H, Hoppe D, Kingsbury D, Ma T. The Game Factory: Using Cooperative Games to Promote Pro-social Behaviour Among Children. Australian Journal of Educational and Developmental Psychology. 2004;(4):97-109.

26. Garaigordobil M. Conducta prosocial: el papel de la cultura, la familia, la escuela y la personalidad. Revista Mexicana de Investigación en Psicología. 2017;6(2):146-157.

27. Garaigordobil M. Juego cooperativo y socialización en el aula: un programa de juego amistoso, de ayuda y cooperación, para el desarrollo socio-afectivo en niños de 6 a 8 años. Seco Olea. 1992;265-288.

28. Garaigordobil M, Berrueco L. Efectos de un programa de intervención en niños de 5 a 6 años: Evaluación del cambio proactivo en factores conductuales y cognitivos del desarrollo. Summa Psicólogica UST. 2007;4(2):3-20.

29. Beltrán O. Efectos de un Programa de Juegos Cooperativos Sobre las Conductas Prosociales y Disociales de Escolares con Problemas de Contravención al Manual de Convivencia [disertación]. Pereira: Universidad Tecnológica de Pereira; 2007.

30. Rebollo-Goñi E. Juegos cooperativos para el fomento de conductas prosociales en la prevención del acoso escolar [disertación]. Logroño: Universidad Internacional de la Rioja; 2013.

31. Osornio L. Juegos Cooperativos como Proyecto de Intervención para Establecer una Mejora de Convivencia Escolar, Paz Y Armonía: Descripción de una Experiencia en una Escuela Telesecundaria de Aculco. Ra Ximhai. 2016;12(3):415-431.

32. Garaigordobil M. Intervención psicológica en la conducta agresiva y antisocial con niños. Psicothema. 2004;16(3):429-435.
33. Tamara M. Los Juegos Cooperativos y su relación con el desarrollo de Habilidades Sociales en la Educación Inicial - Un estudio de casos [disertación]. Buenos Aires: Universidad Abierta Interamericana; 2012.

34. Kerlinger F. Lee H.B. Investigación del comportamiento. Métodos de investigación en ciencias sociales. Cuarta edición. México: McGraw-Hill; 2002.

35. De Minzi, MR. Influencia del modelado de los padres sobre el desarrollo del razonamiento prosocial en los/las niños/as. Interamerican Journal of Psychology. 2009;43(1):187-198.

36. Carlo G, Eisenberg N, Knight GP. An objective measure of adolescents' prosocial moral reasoning. Journal of Research on Adolescence. 1992;2(4):331-349.

37. Mestre V, Frías D, Samper P, Tur A. Adaptación y validación en población española del PROM: una medida objetiva del razonamiento moral prosocial. Acción psicológica. 2002;1(3):221-232.

38. Colombia. Ministerio de Salud. Resolución 8430 de 1993 por la cual se establecen las normas científicas, técnicas y administrativas para la investigación en salud. Bogotá; 1993.

39. Hinnant J, Nelson J, O'Brien M, Keane S, Calkins S. The interactive roles of parenting, emotion regulation and executive functioning in moral reasoning during middle childhood. Cognition \& Emotion. 2013;27(8):1460-1468. 